\title{
Discovery of hybrid $\gamma$ Dor and $\delta$ Sct pulsations in BD+18 4914 through MOST spacebased photometry
}

\author{
J.F. Rowe ${ }^{1}$, J.M. Matthews ${ }^{1}$, C. Cameron ${ }^{1}$, D.A. Bohlender ${ }^{7}$, \\ H. King ${ }^{1}$, R. Kuschnig ${ }^{1}$, D.B. Guenther ${ }^{2}$, A.F.J. Moffat ${ }^{3}$, S.M. Rucinski ${ }^{4}$, \\ D. Sasselov ${ }^{5}$, G.A.H. Walker ${ }^{1}$, W.W. Weiss ${ }^{6}$ \\ ${ }^{1}$ Department of Physics and Astronomy, University of British Columbia \\ 6224 Agricultural Road, Vancouver BC V6T $1 Z 1$ \\ 2 Department of Astronomy and Physics, St. Mary's University \\ Halifax, NS B3H 3C3, Canada \\ 3 Département de physique, Université de Montréal \\ C.P. 6128, Succ. Centre-Ville, Montréal, QC H3C 3J7, Canada \\ ${ }^{4}$ David Dunlap Observatory, University of Toronto \\ P.O. Box 360, Richmond Hill, ON L4C 4Y6, Canada \\ ${ }^{5}$ Harvard-Smithsonian Center for Astrophysics \\ 60 Garden Street, Cambridge, MA 02138, USA \\ ${ }^{6}$ Institut für Astronomie, Universität Wien \\ Türkenschanzstrasse 17, A-1180 Wien, Austria \\ 7 National Research Council of Canada, Herzberg Institute of Astrophysics, \\ 5071 West Saanich Road, Victoria, BC V9E 2E7, Canada
}

\begin{abstract}
We present a total of 57 days of contiguous, high-cadence photometry (14 days in 2004 and 43 in 2005) of the star BD+18 4914 obtained with the MOST ${ }^{1}$ satellite. We detect 16 frequencies down to a signal-to-noise of 3.6 (amplitude $\sim 0.5 \mathrm{mmag}$ ). Six of these are less than 3 cycles/day, and the other ten are between 7 and 16 cycles/day. We intrepret the low frequencies as g-mode $\gamma$ Doradus-type pulsations and the others as $\delta$ Scuti-type p-modes, making BD+18 4914 one of the few known hybrid pulsators of its class. If the g-mode pulsations are high-overtone non-radial modes with identical low degree $\ell$, we can assign a unique mode classification of $n=\{12,20,21,22,31,38\}$ based on the frequency ratio method.
\end{abstract}

\footnotetext{
${ }^{1}$ MOST is a Canadian Space Agency mission, operated jointly by Dynacon, Inc., and the Universities of Toronto and British Columbia, with assistance from the University of Vienna.
} 


\section{Introduction}

$\gamma$ Doradus stars pulsate with typical periods of about 0.8 days (Kaye et al. 1999), consistent with high-overtone nonradial g-modes. They represent one of the newest classes of pulsating variable stars, and about half of the currently known $\gamma$ Doradus stars lie within the $\delta$ Scuti instability strip (Handler 2005). The $\delta$ Scuti variables exhibit p-modes of low radial order, seen only in low degree photometrically, with typical periods of a few hours. Handler \& Shobbrook (2002) have shown that the pulsation characteristics of the two classes can be clearly separated by their values of the pulsation constant $Q$.

The overlap in physical properties of $\gamma$ Doradus and $\delta$ Scuti stars suggested the possibility that hybrid pulsators may exist. The astroseismic implications are exciting since the g-modes would probe the deep interior of the star and the p-modes, its envelope. This has lead to photometric monitoring of $\gamma$ Doradus and $\delta$ Scuti stars to search for hybrid behaviour. The first such hybrid to be discovered was in the binary system HD 209295 by Handler et al. (2002), from careful monitoring of $26 \gamma$ Doradus variables (Handler \& Shobbrook 2002) but the $\gamma$ Doradus pulsations in the primary component are likely caused by tidal interactions with the secondary. The first convincing case of a single hybrid star, the Am star HD 8801, was discovered by Henry \& Fekel (2005) from monitoring 39 stars from a volume-limited sample of $114 \gamma$ Doradus candidates. HD 8801 shows frequencies clustered around 3, 8 and 20 cycles/day (c/d). There were only two frequencies in the $\gamma$ Doradus range and 4 frequencies classified as $\delta$ Scuti in nature. The low number of frequencies makes this star a challenging subject for asteroseismic modeling, but just the existence of a hybrid single star, and the chemical peculiarity of HD 8801, point to new and interesting astrophysics.

We present photometry of the star BD $+184914\left(\alpha=22^{h} 02^{m} 38^{s}, \delta=\right.$ $+18^{\circ} 54^{\prime} 03^{\prime \prime}$ [J2000], $\mathrm{V}=10.6, \mathrm{~B}=11.1$ ) $^{2}$ by the MOST (Microvariability \& Oscillations of STars) satellite in which we detect frequencies consistent with hybrid pulsations.

\section{Photometry}

MOST (Walker, Matthews et al. 2003) is a microsatellite housing a $15-\mathrm{cm}$ telescope feeding a CCD photometer through a custom broadband optical filter. Launched in June 2003 into an 820-km circular Sun-synchronous polar orbit (period $=101.413 \mathrm{~min}$ ), MOST can monitor stars in its Continuous Viewing Zone (CVZ) for up to 8 weeks without interruption. It collects photometry in

\footnotetext{
${ }^{2}$ This research has made use of the SIMBAD database, operated at CDS, Strasbourg, France.
} 
three ways: (1) Fabry Imaging, projecting an extended image of the telescope pupil illuminated by a bright target (see Matthews et al. 2004; Reegen et al. 2005); (2) Guide Star photometry, based on onboard processing of faint stars used for telescope pointing (see Walker et al. 2005); and (3) Direct Imaging, where defocused star images (FWHM $\sim 2.5$ pixels) are projected onto an open area of the Science CCD. This last technique was used to obtain the BD+18 4914 photometry, and details about the Direct Imaging process and reduction are provided by Rowe et al. (2006b); hereafter, RMSK.

The observations of BD+18 4914 were carried out during 14-30 August 2004 and 1 Aug - 15 Sept 2005, for a total of 57 days, during Direct Imaging photometry of the transiting exoplanet system HD 209458 (RMSK). Panel D of Figure 1 shows the 2005 observations of BD+18 4914 using 40-min bins to clearly demonstrate the low-frequency pulsations with periods around 1 day.

The exposure time was $1.5 \mathrm{sec}$, sampled once every 10 seconds. The 2005 data have a raw duty cycle of $97.3 \%$, with about 360000 measurments in 43 days. After rejection of points with extreme cosmic ray activity and other obvious outliers, the net duty cycle is $75 \%$. The 2004 data have a net duty cycle of $70.4 \%$. The combined data set have a total of 443154 measurements in 57 days. There are gaps about 30 minutes long during each 101.4 minute orbit of the satellite. The data do not suffer from the cycle/day aliases common to groundbased photometry. They have only minor alias sidelobes at $14.2 \mathrm{c} / \mathrm{d}$ (see the spectral window function in Figure 1) which do not lead to any ambiguities in the frequency identifications.

The photometric reduction scheme is described in RMSK for the 2004 data set. A paper on the data of 2005 is in preparation. After dark and flatfield corrections, photometry is obtained by a combination of aperture measurments for the core of the stellar point spread function (PSF) and a fit to the Moffatprofile (Moffat 1969) model for the wings of the PSF.

The observations are made through a single broadband filter $(350-750 \mathrm{~nm})$ made specifically for the MOST mission, which has about $3 \times$ the throughput of a Johnson $\mathrm{V}$ bandpass but is not tied to any standard photometric system.

\section{Frequency Analysis}

A preliminary analysis of the 2004 observations of BD+18 4914 was presented in Rowe et al. (2006a) that showed the dual nature of its pulsations. Here we take a more methodological approach to the frequency analysis.

We begin by computing the discrete Fourier transform (DFT) of the 2005 time series. The amplitude spectrum is shown in panel A of Figure 1. The data 

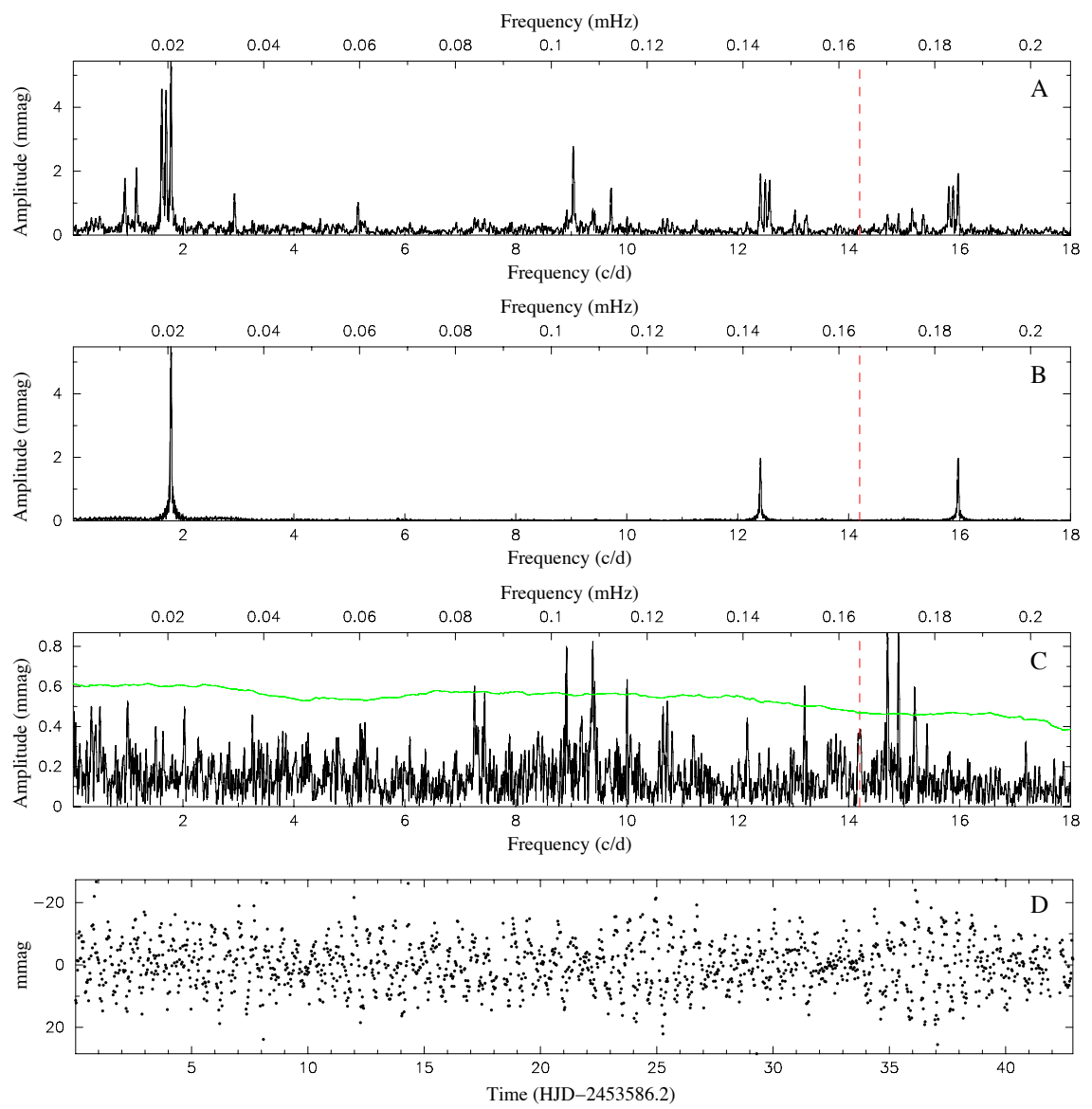

Figure 1: Panel A shows the amplitude spectrum of the 2005 data set. Panel B shows the corresponding window function for the highest peak in Panel A. Panel $\mathrm{C}$ shows the amplutude spectrum after prewhitening of the 8 strongest frequencies. The detection limit of 3.6 times the noise is also shown. (The MOST orbital frequency is marked as a dashed vertical line.) Panel D presents the photometric data in 40-min bins to highlight the low-frequency $(2 \mathrm{c} / \mathrm{d})$ oscillations.

are then fitted using an equation of the form

$$
m a g=A_{0}+\sum_{j=1, n} A_{j} \cos \left(2 \pi f_{j} t+\phi_{j}\right),
$$

Where $A_{0}$ is a linear offset and $f_{j}, A_{j}$ and $\phi_{j}$ are the frequency, amplitude and phase for each successive peak found in the amplitude spectrum using the 
Leveberg-Marqardt approach (Press et al. 1992). After each fit, the DFT of the residuals is recalculated and the next largest amplitude is chosen, but only if the Signal-to-Noise $(\mathrm{S} / \mathrm{N})$ is greater than 3.6. The $\mathrm{S} / \mathrm{N}$ is defined as the amplitude of the peak in the spectrum divided by the mean of nearby frequencies. We use a window about $3 \mathrm{c} / \mathrm{d}$ wide in frequency space, centred on the highest peak, to calculate the mean which we use as an estimate of the local noise floor.

We detect 16 frequencies in the 2005 photometry with a S/N greater than 3.6. Our results and best fit parameters are listed in Table 1.

If the same procedure is repeated for the 2004 photometry, then the relatively short duration (14 days) of the data set causes degeneracies in the nonlinear solution because of poor frequency resolution. Specifically, the solutions for frequencies $j=\{2,3\}$ in Table 1 converge to identical values with phases offset by $\pi$ radians. The amplitudes in turn grow unreasonably large. The problem is illustrated in Figure 2. The two top panels of Figure 2 compare the amplitude spectra of the 2005 and 2004 data sets in the frequency range $0.1-3 \mathrm{c} / \mathrm{d}$. The 2005 data set has a higher frequency resolution due to its longer duration. The 2004 amplitude spectrum shows the same three peaks as seen in 2005, but the amplitudes of the peaks appear to be larger in amplitude. This is due to the degeneracy of the solution at these low frequencies in the shorter time series. The two bottom panels of Figure 2 compare the DFT at a higher frequency range, $8-11 \mathrm{c} / \mathrm{d}$. The frequency and amplitude changes seen in the low frequency range are no longer apparent.

To avoid the degeneracy problem, the frequency solution from the 2005 dataset is used as the initial solution for the non-linear routine applied to the 2004 data, with the frequencies held as fixed parameters to derive the amplitudes to be determined. If one examines the DFT of the residuals from the best fit there are no significant peaks remaining, thus the 2005 frequencies solution is valid for the 2004 data set. The best fit parameters are presented in column 5 of Table 1 . This does not imply that the frequencies are constant from 2004 to 2005 , only that the 2004 data set is too short to give meaningful results for a a change in frequeny in the low frequency regime.

To estimate the errors in our fitted parameters, we perform a "bootstrap" analysis. This method involves redetermining the fitted parameters with randomly generated data sets. The new datasets are created by randomly selecting data from the original time series with replacement. In other words, any individual data point can be chosen more than once but the total number of selected points is always the same as the original data set. The bootstrap method is effective since it preserves the same noise profile in each random set as exists in the original data and given enough iterations will produce error distributions for each fitted variable. For further demonstrations and discussion of the bootstrap method, we refer the reader to Cameron et al. (2006) and Saio et al. (2006). 

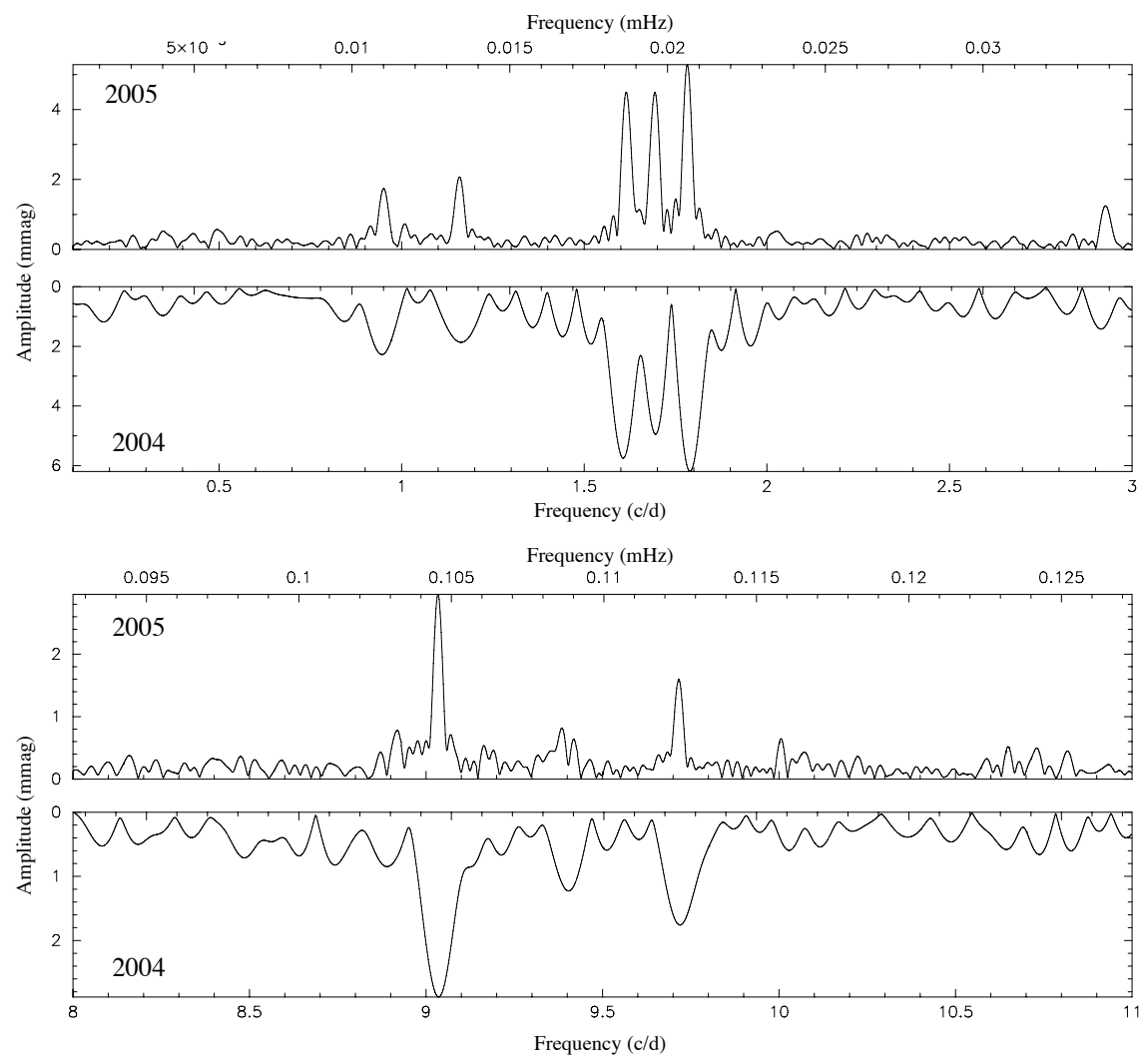

Figure 2: Comparisons of the 2004 and 2005 Fourier amplitude spectra. The two top panels compare the two data sets in the frequency range $0-3 \mathrm{c} / \mathrm{d}$. The two bottom panels cover the range $8-11 \mathrm{c} / \mathrm{d}$.

We generated 22083 and 18595 bootstrap iterations for the 2004 and 2005 data sets, respectively. The $1-\sigma$ error distributions using a Gaussian model are given in Table 1 for both data sets.

\section{A hybrid pulsator}

The frequencies found in BD+18 4914 cluster in the two ranges typical of $\gamma$ Dor and $\delta$ Sct oscillation modes, making this a clear candidate for a hybrid pulsator.

We can quantify this assessment using the criterion established by Handler \& Shobbrook (2002) that the pulsation constant Q distinguishes the $g-$ and $p-$ 


\begin{tabular}{ccccccc}
\hline \hline$j$ & $f_{j}(\mathrm{c} / \mathrm{d})$ & $A_{j}(\mathrm{mmag})$ & $\phi_{j}(\mathrm{rad})$ & $A_{j}^{2004}(\mathrm{mmag})$ & $\mathrm{S} / \mathrm{N}$ & $\mathrm{Q}$ (days) \\
& $\sigma_{f_{j}}$ & $\sigma_{A_{j}}$ & $\sigma_{\phi_{j}}$ & $\sigma_{A_{j}^{2004}}$ & & \\
\hline 6 & 0.9496 & 1.97 & 6.08 & 1.65 & 10.0 & 0.34 \\
& 0.0004 & 0.06 & 0.07 & 0.10 & & \\
5 & 1.1586 & 2.07 & 3.60 & 1.75 & 9.9 & 0.28 \\
& 0.0004 & 0.06 & 0.07 & 0.10 & & \\
2 & 1.6150 & 4.74 & 0.55 & 4.57 & 19.0 & 0.20 \\
& 0.0002 & 0.06 & 0.03 & 0.10 & & \\
3 & 1.6924 & 4.60 & 2.05 & 4.48 & 20.0 & 0.19 \\
& 0.0002 & 0.06 & 0.03 & 0.10 & & \\
1 & 1.7829 & 5.25 & 5.99 & 4.98 & 19.4 & 0.18 \\
& 0.0002 & 0.06 & 0.03 & 0.10 & & \\
8 & 2.9286 & 1.22 & 3.08 & 0.87 & 7.0 & 0.11 \\
& 0.0007 & 0.06 & 0.12 & 0.10 & & \\
14 & 7.2530 & 0.65 & 0.22 & 1.04 & 3.9 & 0.04 \\
& 0.0012 & 0.06 & 0.21 & 0.10 & & \\
15 & 7.4354 & 0.53 & 0.03 & 0.42 & 3.8 & 0.04 \\
& 0.0015 & 0.06 & 0.27 & 0.10 & & \\
11 & 8.9122 & 0.84 & 4.66 & 0.94 & 4.9 & 0.04 \\
& 0.0010 & 0.06 & 0.17 & 0.10 & & \\
4 & 9.0348 & 3.09 & 0.43 & 3.08 & 16.3 & 0.04 \\
& 0.0003 & 0.06 & 0.05 & 0.10 & & \\
12 & 9.3847 & 0.84 & 0.90 & 1.10 & 5.1 & 0.03 \\
& 0.0010 & 0.06 & 0.17 & 0.10 & & \\
7 & 9.7156 & 1.56 & 3.95 & 1.85 & 9.4 & 0.03 \\
\multirow{4}{*}{13} & 0.0005 & 0.06 & 0.09 & 0.10 & & \\
& 10.0043 & 0.60 & 3.09 & 0.50 & 3.9 & 0.03 \\
& 0.0013 & 0.06 & 0.23 & 0.10 & & \\
& 14.6977 & 0.91 & 3.07 & 0.64 & 7.0 & 0.02 \\
& 0.0010 & 0.06 & 0.16 & 0.10 & & \\
& 14.8967 & 0.87 & 3.09 & 0.85 & 6.9 & 0.02 \\
& 0.0010 & 0.06 & 0.16 & 0.10 & & \\
& 15.4106 & 0.46 & 3.76 & 0.24 & 3.8 & 0.02 \\
& 0.0018 & 0.06 & 0.31 & 0.09 & & \\
\hline \multirow{4}{*}{10} & & & & & &
\end{tabular}

Table 1: Observed frequencies and parameters for $\mathrm{BD}+18$ 4914. The epoch is $H J D=2453586.20349121$. 
modes in this type of star. It was shown that although the pulsation periods of $\delta$ Scuti and $\gamma$ Doradus overlap there is a clear separation when $Q$ is considered (see Figure 9 of Handler \& Shobbrook (2002)). To calculate Q, we require basic properties of the star: $\log g, M_{b o l}$ and $T_{\text {eff }}$. We obtained a $10 \AA / \mathrm{mm}$ spectrum with the $1.8 \mathrm{~m}$ Plasket telescope at the Dominion Astrophysical Observatory ${ }^{3}$

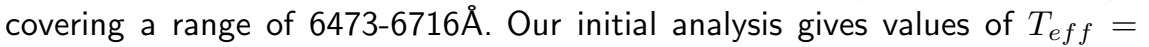
$7250 \mathrm{~K}, \log g=3.7 \mathrm{cgs}$ and $M_{b o l}=2.5$. Using Equation 1 from Handler \& Shobbrook (2002), we compute values of $Q$ for each frequency, and they are presented in Table 1.

With analogy to the Am star hybrid pulsator HD 8801 (Henry \& Fekel 2005), we assume that all frequencies less than $3.0 \mathrm{c} / \mathrm{d}$ are of $\gamma$ Doradus type and the frequencies higher than $6 \mathrm{c} / \mathrm{d}$ are of $\delta$ Scuti type. Although we do not have enough information (multibandpass photometry or spectral line profile variation data) to make pulsation mode identifications, we can apply the Frequency Ratio Method (FRM) described by Moya et al. (2005) to the 6 lowest frequencies. This assumes that the observed $\gamma$ Dor pulsations can be described by the asymptotic approximation under the assumption of adiabaticity and spherical symmetry (Tassoul 1980). If the modes all share the same degree $\ell$, then the ratio of the frequencies can be approximated by

$$
\frac{\sigma_{\alpha 1}}{\sigma_{\alpha 2}} \approx \frac{n_{2}+1 / 2}{n_{1}+1 / 2}
$$

Under these assumptions, we have searched for 6 overtone $n$ values which satisfy Equation 2, taking an error of $\pm 1.3 \times 10^{-2}$ for calculation of the sets of possible overtones (Suárez et al. 2005). Restricting our search to overtones up to and including $n=60$, we find only one viable solution: $n=\{12,20,21,22,31$, and 38$\}$ for the frequencies labeled $j=\{8,1,3,2,5$, and 6$\}$ in Table 1 . If we search up to $n=100$, then the density of natural number ratios compared to our error bounds allows for 78 more solutions. Regardless, other mode identification methods need to be applied to restrict the possible values of degree $\ell$.

Observations of other $\gamma$ Doradus pulsators have shown that the amplitudes can be variable, such as seems to be the case with 9 Aurigae (see Kaye et al. 1997 and references therein). Using our results for the best-fit parameters of the 2004 and 2005 photometry, we can examine the possibility of amplitude changes over a 1-year interval. In Figure 3 we plot the measured amplitudes from 2004 versus those from 2005 (see Table 1 ) with $1 \sigma$ error bars. No significant amplitude changes have occurred. We define detection of an amplitude change

\footnotetext{
${ }^{3}$ Based in part on observations obtained at the Dominion Astrophysical Observatory, Herzberg Institute of Astrophysics, National Research Council of Canada
} 
as

$$
\frac{\Delta A_{j}}{\sigma}=\frac{A_{j}-A_{j}^{2004}}{\sqrt{\sigma_{A_{j}}^{2}+\sigma_{A_{j}^{2004}}^{2}}}
$$

where the definitions are same as presented in Table 1 . The distribution of $\Delta A_{j} / \sigma$ appears to be non-Gaussian. We can test this with an student T-test and an F-test. To do so, we generated 10000 sets of 16 random Gaussian deviates and calculated the student T-test probability and F-test probability for the distributions to have similar means to our $\Delta A_{j} / \sigma$ sample. Our adapted criteria (for the samples to differ) is a probability less than $0.0026(3 \sigma)$. For the T-test, none of the 10000 sets were rejected; thus the means are statistically similar. For the F-test, $26.7 \%$ of cases were rejected (i.e., only $27.6 \%$ produced a probability less than 0.0026 ). At our chosen $3 \sigma$ threshold, our sample has a Gaussian distribution thus an amplitude change is not statistically significant. If we apply the tests to our 2004 and 2005 amplitude distributions and ask if the two distributions differ, then the student T-test gives a probability of $90.9 \%$ and the F-test, a probability of $88.3 \%$ that the two have similar means and variances, respectively.

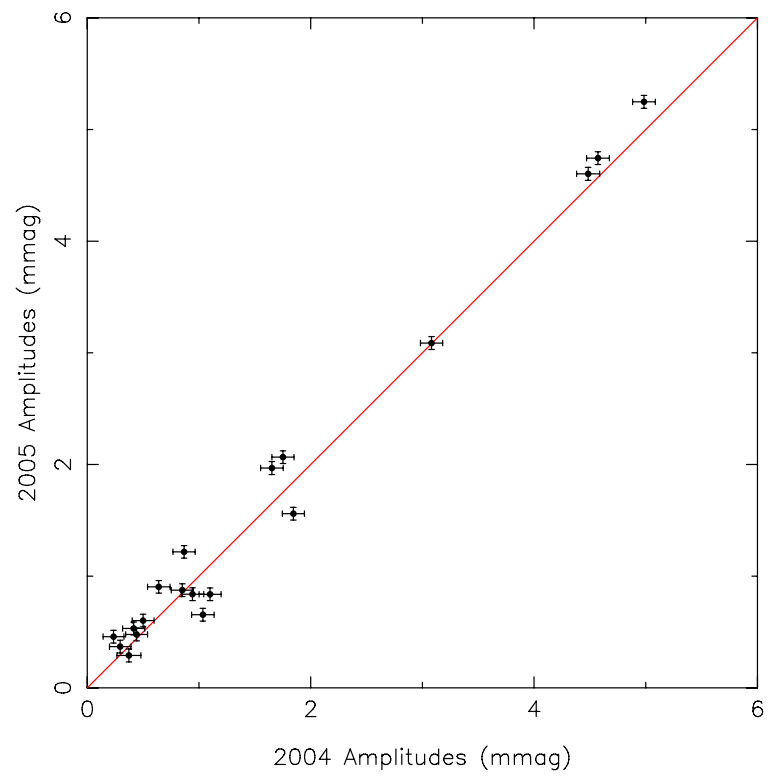

Figure 3: The measured amplitudes for the 2004 and 2005 observation campaigns plotted against each other, with $1 \sigma$ error bars. 


\section{Conclusions}

We have presented 14 and 43 days of nearly continuous photometry obtained by the MOST satellite. From this photometry, we detect 16 frequencies whose amplitudes have $S / N>3.6$, clustered in two ranges consistent with $\gamma$ Doradustype and $\delta$ Scuti-type pulsations. With 6 frequencies in the $\gamma$ Doradus range, application of the FRM method assuming a common degree $\ell$ yields a unique set of radial orders of the pulsations. Comparison of the 2004 and 2005 data sets show no statistically significant changes in the pulsation amplitudes. However, this star is scheduled to be observed for a third time by MOST in the fall of 2006 to gain insight into the stability of the observed frequencies and remove any degeneracies from our fits. Groundbased spectroscopy and multicolour photometry will be necessary to obtain independent mode identifications to confirm whether the FRM assumptions we have made are valid and to take advantage of the potential of $\mathrm{BD}+184914$ for asteroseismology.

Acknowledgments. The contributions of JMM, DBG, AFJM, SR, and GAHW are supported by funding from the Natural Sciences and Engineering Research Council (NSERC) Canada. RK is funded by the Canadian Space Agency. WWW received financial support from the Austrian Science Promotion Agency (FFG - MOST) and the Austrian Science Funds (FWF - P17580).

\section{References}

Cameron, C., et al. 2006, CoAst, 148, 34

Handler, G. \& Shobbrook, R.R. 2002, MNRAS, 333, 251

Handler, G., et al. 2002, MNRAS, 333, 262

Handler, G. 2005, JApA, 26, 241

Henry, G.W. \& Fekel, F.C. 2005, AJ, 129, 2026

Kaye, A. B., et al. 1997, DSSN, 11, 32

Kaye, A. B., et al. 1999, PASP, 116, 558

Matthews et al. 2004, Nature, 430, 51

Moffat, A.F.J. 1969, A\&A , 3, 455

Moya, A. et al. 2005, A\&A , 432, 189

Press, W.H. et al. 1992, Numerical Recipes in FORTRAN 77 (2nd ed, Cambridge:

Cambridge Univ. Press)

Reegen, P., et al. 2005, MNRAS, 367, 1417

Rowe, J.F., et al. 2006a, Mm. S.A.It., 77, 282

Rowe, J.F., et al. 2006b, ApJ , 646, 1241 (RMSK)

Saio, H., et al. 2006, astro-ph/0606712

Suárez et al. 2005, A\&A , 443, 271

Tassoul, M. 1980, ApJ, 43, 469

Walker, G.A.H., Matthews, J.M., et al. 2003, PASP, 115, 1023

Walker, G.A.H., et al. 2005, ApJ, 635, 77 